\title{
Blickdiagnose
}

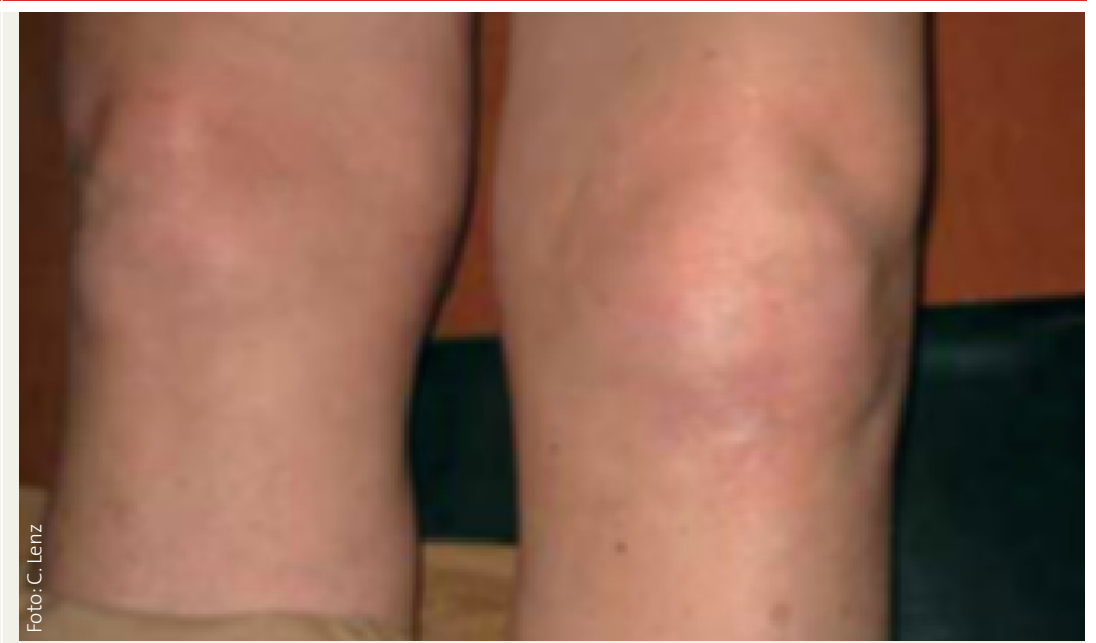

\section{SAPHO und das Knie}

Eine 63-jährige Patientin mit bekannter Psoriasis berichtet über eine seit vier Monaten rezidivierend auftretende Schwellung und Rötung des linken Knies und Unterschenkels.

Klinisch zeigt sich eine diskrete Schwellung und leichte Verfärbung der Haut, radiologisch eine starke Periostreaktion der linken Tibia. Im MRT kann ein pathologisches Knochenmarködem in der Tibia, eine periostale Verdickung und ein angrenzendes Weichteilödem mit deutlicher Kontrastmittelaufnahme nachgewiesen werden, am ehesten mit dem Bild einer Osteitis vereinbar. Szintigraphisch imponiert eine massive Speicherung der Tibia.

Aufgrund dieser Befunde wird der dringende Verdacht eines SAPHO-Syndroms geäußert. Dabei handelt es sich um eine den seronegativen Spondarthritiden zuordenbare Knochenentzündung ohne überdurchschnittliche HLA-B-27-Prävalenz, die durch folgende Symptome charakterisiert ist: Synovitis, Akne, palmoplantare Pustulose, Hyperostosis und Osteitis.

Die genannten Symptome müssen nicht gleichzeitig vorkommen, weshalb das Syndrom wahrscheinlich seltener diagnostiziert wird, als es tatsächlich vorliegt. Betroffen sind häufig die so genannten stammnahen Gelenke (z. B. Sternoclaviculargelenk), wobei noch keine genauen Angaben bezüglich der Ursache und Häufigkeit vorliegen. Zwar ist eine Pustelbildung in den Handinnenflächen sowie unter den Fußsohlen richtungsweisend, jedoch sollte der Verdacht immer entstehen, wenn pustulöse Hauterkrankungen, verbunden mit „rheumatischen“ Beschwerden, auftreten.

Da verschiedene Krankheitsverläufe vorkommen - bislang noch ohne standardisierte Therapiekonzepte - wird die Behandlung symptomatisch nach rheumatischen Gesichtspunkten ausgerichtet.

- Dr. Christine Lenz, Fachärztin für Allgemeinmedizin, Isaraustraße 13, D-82538 Geretsried

Keyword: SAPHO syndrome

\section{Ihr besonderer Fall?}

Sicher sehen auch Sie ab und an einen besonders eindrucksvollen Befund in Ihrer Praxis. Fotografieren Sie ihn, schreiben Sie uns unter dem Stichwort Blickdiagnose, bei Veröffentlichung erhalten Sie 100 Euro.

MMW-Fortschritte der Medizin

E-Mail: manhart@urban-vogel.de

Fax: 089/4372-1420

Weitere interessante Blickdiagnosen finden Sie in unserem Online-Archiv unter www.mmw.de. 